\title{
Influência da luz e da temperatura na germinação de sementes de Marcetia taxifolia (A. St.-Hil.) DC. (Melastomataceae)
}

\author{
Fernando A.O. Silveira ${ }^{1}$, Daniel Negreiros ${ }^{1}$ e G. Wilson Fernandes ${ }^{1,2}$
}

Recebido em 05/02/2003. Aceito em 11/05/2004

\begin{abstract}
RESUMO - (Influência da luz e da temperatura na germinação de sementes de Marcetia taxifolia (A. St.-Hil.) DC. (Melastomataceae)). Marcetia taxifolia (A. St.-Hil.) DC. (Melastomataceae) é um arbusto amplamente distribuído nos campos rupestres da Serra do Cipó, Brasil. É uma espécie com potencial na recuperação de áreas degradadas e este estudo apresenta os primeiros dados sobre o efeito da luz e da temperatura na germinação de suas sementes. Sementes foram coletadas em maio/2001 e foram submetidas a testes de germinação nas temperaturas constantes de 15, 20, 25, 30 e $35^{\circ} \mathrm{C}$ sob fotoperíodo de 12 horas e em ausência de luz durante 30 dias. Foram encontradas diferenças significativas entre a percentagem de germinação e tempo médio de sementes submetidas ao tratamento claro e escuro a 15, 20, 25 e $30^{\circ} \mathrm{C}$ ( $<<0,05$; todos). Sob a temperatura de $35^{\circ} \mathrm{C}$, a germinação foi completamente inibida. As maiores percentagens de germinação foram obtidas a 15 e $20^{\circ} \mathrm{C}$, as quais foram superiores a 25 e $30^{\circ} \mathrm{C}$ (ANOVA = 13,65; p < 0,001). Os dados contribuem para estudos posteriores que visem a propagação da espécie com fins de reabilitação ambiental.
\end{abstract}

Palavras-chave: campo rupestre, fotoblastismo, germinação de sementes, Serra do Cipó

\begin{abstract}
Light and temperature influence on seed germination of Marcetia taxifolia (A. St.-Hil.) DC. (Melastomataceae)). Marcetia taxifolia (A. St.-Hil.) DC. (Melastomataceae) is a shrub widely distributed in the rupestrian fields of Serra do Cipó, Brazil. In spite of M. taxifolia being a potential species to be used in land rehabilitation, no complete study has been conducted regarding its seed germination. The goal of this work was to evaluate the effect of light and temperature on seed germination of M. taxifolia. Seeds were collected in May/2001 and were submitted to germination tests at constant temperatures of $15,20,25,30$ and $35^{\circ} \mathrm{C}$ under a photoperiod of 12 hours, and in the absence of light for 30 days. There were statistically significant differences between seed germination and mean time of germination for seeds submitted to the light and dark treatments at 15, 20, 25 and $30^{\circ} \mathrm{C}\left(\mathrm{p}<0,05\right.$; all). Seed germination under $35^{\circ} \mathrm{C}$ was totally inhibited. The higher germination percentages were obtained at 15 and $20^{\circ} \mathrm{C}$, which differed significantly from the temperatures of 25 and $30^{\circ} \mathrm{C}$ $($ ANOVA $=13,65 ; \mathrm{p}<0,001)$. The data contribute to further studies focusing the propagation of M. taxifolia for land rehabilitation purposes.
\end{abstract}

Key words: rupestrian fields, photoblastism, seed germination, Serra do Cipó

\section{Introdução}

O cerrado é um dos biomas mais ricos e ameaçados do planeta, representando uma das áreas prioritárias para conservação da biodiversidade (Lara \& Fernandes 1996; Myers et al. 2000). O campo rupestre é uma das fisionomias mais críticas (Costa et al. 1998) e na Serra do Cipó (MG), ocorre em regiões com altitude superior a $1.000 \mathrm{~m}$, onde predominam afloramentos rochosos de quartzito. Sua flora predominante esclerófila apresenta espécies com alto grau de endemismo (Giulietti et al. 1987; Mendonça \& Lins 2000).

As plantas dos campos rupestres da Serra do Cipó estão adaptadas a condições extremas, tais como alta insolação, solos rasos, secos e nutricionalmente pobres, além das temperaturas que variam grandemente ao longo do ano (Giulietti et al. 1987). Devido ao alto grau de impactos nesta região (extração de madeiras, de flores, ação do fogo e loteamentos), estudos são necessários sobre a biologia básica das espécies vegetais, uma vez que várias destas encontram-se ameaçadas de extinção (Mendonça \& Lins 2000).

Poucos estudos auto-ecológicos e de biologia reprodutiva têm sido realizados em campos rupestres, embora sejam de fundamental importância para implementação de estratégias de conservação (Madeira \& Fernandes 1999; Jacobi et al. 2000). Estudos sobre germinação de sementes de plantas são cruciais neste contexto (Melo et al. 1998; Gomes et al. 2001; Ranieri et al. 2003). Além disto, o conhecimento da biologia das sementes é fundamental para o entendimento do

\footnotetext{
1 Ecologia Evolutiva de Herbívoros Tropicais/DBG, Instituto de Ciências Biológicas, Universidade Federal de Minas Gerais, C. Postal 486, CEP 30161-970, Belo Horizonte, MG, Brasil

2 Autor para correspondência: gwilson@icb.ufmg.br
} 
estabelecimento de plântulas, sucessão, regeneração natural e reabilitação de áreas degradadas (VásquesYanes \& Orozco-Segovia 1993; Gomes \& Fernandes 2002).

Dentre os principais fatores que afetam a germinação das sementes, merecem destaque a temperatura e a luz (Labouriau 1983). A temperatura pode regular a germinação por três maneiras: determinando a capacidade e taxa de germinação; removendo a dormência primária ou secundária; e induzindo dormência secundária (Bewley \& Black 1994). Garcia \& Diniz (2003) verificaram que as sementes de espécies de Vellozia apresentam alta germinabilidade em altas temperaturas, enquanto que Ferreira et al. (2001) verificaram que para várias espécies de Asteraceae o percentual de germinação foi maior a $20^{\circ} \mathrm{C}$ do que 25 ou $30^{\circ} \mathrm{C}$. Ranieri et al. (2003) observaram que a temperatura ótima para a germinação das sementes de Lavoisiera cordata da Serra do Cipó (Melastomataceae) é $20^{\circ} \mathrm{C}$. Por sua vez, a luz regula a germinação através da molécula do fitocromo. As sementes que germinam na presença de luz são chamadas fotoblásticas positivas, enquanto aquelas nas quais a germinação é inibida pela luz são chamadas fotoblásticas negativas (Vásques-Yanes \& Orozco-Segovia 1993; Bewley \& Black 1994).

A espécie Marcetia taxifolia (A. St.-Hil.) DC. (Melastomataceae) é representada por arbustos e subarbustos eretos que alcançam até 3,0m alt. M. taxifolia apresenta ampla distribuição geográfica, desde as restingas na costa brasileira até o topo das montanhas mais altas da Venezuela, situadas a $3.000 \mathrm{~m}$ de altitude (Martins, com. pess.). Existem pelo menos duas variedades desta espécie, sendo uma em forma de touceira, com tamanho reduzido, que produz flores roxas e a segunda produz flores brancas e com indivíduos atingindo aproximadamente $2 \mathrm{~m}$ alt. Aparentemente floresce e frutifica ao longo de todo o ano, produzindo grande quantidade de sementes pequenas (Martins, com. pess.). Entretanto, na população em estudo, houve sincronia nas fenofases.

Na Serra do Cipó, os indivíduos de M. taxifolia, além de ocorrer em simpatria com outras espécies de Marcetia (Martins, com. pess.), apresentam algumas características peculiares em relação a populações de outras regiões. Apresenta-se na forma de touceira, destacando-se a presença do xilopódio, que não é observado em plantas da restinga, florescem em setembro e frutificam em novembro, Além disso, possuem tamanho bem reduzido quando comparado a indivíduos de outras regiões (Vale, com. pess.). Apesar de ser espécie de ampla distribuição geográfica, nenhum estudo de germinação de sementes desta espécie foi realizado. Assim, este trabalho teve como objetivo estudar a influência da luz e da temperatura na germinação de sementes de $M$. taxifolia.

\section{Material e métodos}

A partir de indivíduos de que atingiam até $2 \mathrm{~m}$ alt., de flores brancas, as sementes foram coletadas no mês de maio/2001, na Serra do Cipó, MG, Brasil (19¹7’'S, $43^{\circ} 35^{\prime} \mathrm{W}$ ). O clima desta região é mesotérmico (Cwb na classificação de Köppen), com uma estação seca que dura aproximadamente quatro meses (de maio a agosto) e uma estação chuvosa, de oito meses de duração (de setembro a abril) (Madeira \& Fernandes 1999). A precipitação média anual dos últimos 10 anos gira em torno de $1.350 \mathrm{~mm}$ e a temperatura média anual varia de 25 a $30^{\circ} \mathrm{C}$ no verão, enquanto no inverno a variação da temperatura é de 8 a $18^{\circ} \mathrm{C}$ (Madeira \& Fernandes 1999).

Não foi utilizado processo algum para desinfetar a superfície das sementes e estas foram colocadas imediatamente para germinar. Lotes de sementes foram submetidos a testes de germinação nas temperaturas constantes de $15,20,25,30$ e $35^{\circ} \mathrm{C}$, sob fotoperíodo de 12 horas de luz branca fluorescente e em ausência de luz durante 30 dias. Foram montadas 4 réplicas com 25 sementes em placas de Petri forradas com folha dupla de papel filtro e umedecidas com solução de nistatina (Gomes et al. 2001). As placas-de-petri foram colocadas em câmaras de germinação (BOD) sendo a germinação verificada a cada $24 \mathrm{~h}$. O critério para se considerar a semente como germinada era a ocorrência de protrusão radicular.

O tratamento de ausência de luz foi obtido envolvendo-se as placas de Petri em folhas duplas de papel alumínio. A observação da germinação foi realizada sob luz verde de segurança. O tempo médio de germinação (TM) foi calculado de acordo com Labouriau (1983).

O delineamento experimental foi o totalmente casualizado com 4 repetições de 25 sementes por tratamento. Os valores de percentagem de germinação foram transformados em raiz de arco seno para sua normalização. As médias entre os tratamentos foram comparadas através de análise de variância fatorial seguida do teste de Tukey a 5\% de significância (Zar 1996). 


\section{Resultados e discussão}

As percentagens de germinação de sementes submetidas ao fotoperíodo de $12 \mathrm{~h}$ foram superiores àquelas das sementes submetidas ao escuro contínuo em todas as temperaturas estudadas ( $\mathrm{p}<0,05$; Tab. 1 ). As sementes de $M$. taxifolia podem ser classificadas como fotoblásticas positivas, uma vez que respondem positivamente ao estímulo luminoso. Entretanto, a germinação não foi restrita à presença de luz, uma vez que também ocorreu no escuro contínuo, apesar de significativamente menor. O mesmo padrão foi encontrado em relação ao tempo médio (TM) de germinação. Em todas as temperaturas, o TM foi maior no claro do que no escuro contínuo (Tab. 1). Resultados similares aos deste estudo foram encontrados por Garcia \& Diniz (2003), que verificaram que as pequenas sementes de Vellozia spp. da Serra do Cipó são fotoblásticas positivas, embora altas temperaturas promoveram a germinação no escuro.

Tabela 1. Média da percentagem final de germinação de sementes de Marcelia taxifolia (A. St.-Hil.) DC. e tempo médio de germinação (TM) nas temperaturas de $15,20,25,30$ e $35^{\circ} \mathrm{C}$ sob fotoperíodo de 12 horas e escuro contínuo em maio/2001.

\begin{tabular}{cccccc}
\hline & \multicolumn{3}{c}{ Claro } & & \multicolumn{2}{c}{ Escuro } \\
\cline { 2 - 3 } \cline { 5 - 6 } $\begin{array}{c}\text { Temperatura } \\
\left({ }^{\circ} \mathrm{C}\right)\end{array}$ & $\%$ & T M & & $\%$ & T M \\
\cline { 6 - 7 } 15 & $52^{\mathrm{a}}$ & 32,73 & & $5^{\mathrm{c}}$ & 3,97 \\
20 & $51^{\mathrm{a}}$ & 22,30 & & $7^{\mathrm{c}}$ & 4,50 \\
25 & $30^{\mathrm{ab}}$ & 9,90 & & $5^{\mathrm{c}}$ & 2 \\
30 & $14^{\mathrm{b}}$ & 7,87 & & $3^{\mathrm{c}}$ & 1,77 \\
35 & $0^{\mathrm{c}}$ & 0 & & $0^{\mathrm{c}}$ & 0 \\
\hline
\end{tabular}

Letras diferentes representam médias estatisticamente diferentes entre si pelo Teste de Tukey a 5\% no claro.

A presença de sementes fotoblásticas positivas é bem conhecida na família Melastomataceae (Andrade 1995; Zaia \& Takaki 1998; Baskin et al. 1999, SousaSilva 2001; Valio \& Scarpa 2001). Sementes de espécies desta família que habitam ambientes de dossel fechado geralmente são pequenas e podem permanecer no solo à espera da abertura de clareiras que proporcionam a entrada de luz. Portanto, a luz é um fator limitante para que estas sementes possam germinar e dar origem a novas plântulas (Elisson et al. 1993; Baider et al. 1999).

Em sementes pequenas, a ocorrência de germinação na presença da luz pode ser considerada como uma característica adaptativa. Sementes pequenas geralmente são fotoblásticas positivas (Hewittt 1998) e a incapacidade destas sementes de germinar na ausência de luz faz com que elas o façam apenas nas camadas superficiais do solo, onde a luz pode atingí-las. Logo, se uma semente fotoblástica positiva estiver enterrada, é necessário que a terra seja revolvida para promover a germinação.

A temperatura influenciou a germinação de sementes de $M$. taxifolia no claro. O início da germinação deu-se entre o $6^{\circ}$ e $13^{\circ}$ dia de acordo com a temperatura (Fig. 1). As maiores percentagens de germinação foram obtidas a 15 e $20^{\circ} \mathrm{C}(52 \%$ e $51 \%$ respectivamente, Tab. 1). A germinação apresentou relação inversa com o aumento de temperatura, diminuindo à medida que a temperatura aumentou. Dessa forma, na temperatura de $35^{\circ} \mathrm{C}$, a germinação não ocorreu. Mesmo nas temperaturas mais eficientes para promover a germinação $\left(15\right.$ e $\left.20^{\circ} \mathrm{C}\right)$, a taxa de germinação foi pouco superior a 50\% (Tab. 1), demonstrando baixa germinabilidade. O teste de viabilidade do tetrazólio não foi realizado devido ao pequeno tamanho das sementes.

Sementes de várias espécies do cerrado germinam entre 10 e $45^{\circ} \mathrm{C}$ (Felippe \& Silva 1984). Entretanto, altas temperaturas, como $35^{\circ} \mathrm{C}$, podem reduzir significativamente a taxa de germinação em algumas Melastomataceae como Tibouchina grandifolia, $T$. benthamina e T. moricandiana (Andrade 1995). Também foram observadas alterações na morfologia das plântulas de Maquira sclerophylla (Moraceae) submetidas a $35^{\circ} \mathrm{C}$ (Miranda \& Ferraz 1999). Estas alterações incluem a formação de raiz primária pouco

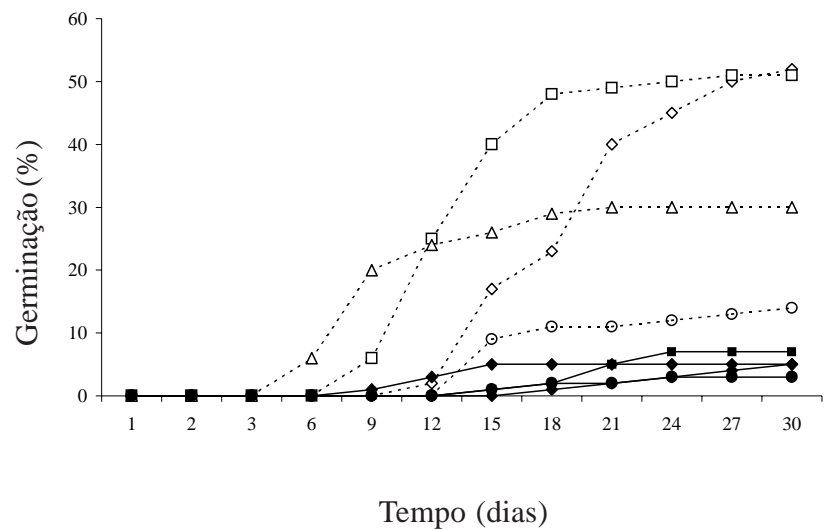

Figura 1. Germinação cumulativa (\%) de sementes de Marcetia taxifolia (A. St.-Hil.) DC. sob fotoperíodo de 12 horas (linha tracejada) e no escuro contínuo (linha contínua), nas temperaturas de $15(\diamond), 20(\square), 25(\triangle)$ e $30^{\circ} \mathrm{C}(\bigcirc)$ em maio de 2001. Belo Horizonte, MG, maio/2004. 
desenvolvida e com extremidade necrosada, pouca ou nenhuma raiz secundária, epicótilo atrofiado, eófilos reduzidos e/ou necrosados. Por outro lado, Garcia \& Diniz (2003) verificaram que altas temperaturas favorecem a germinação de sementes de Vellozia spp.

No tratamento de escuro contínuo, a germinação foi baixa em todas as temperaturas e não houve diferenças significativas entre as taxas de germinação de sementes submetidas entre diferentes temperaturas no escuro contínuo ( $p>0,05)$.

As plantas de campos rupestres podem ocorrer em solos arenosos e em afloramentos rochosos. No entanto, em afloramentos quartzíticos, as populações de $M$. taxifolia apresentam baixa densidade (F. Vale, com. pess.). É possível que o fator limitante para o estabelecimento de novos indivíduos em afloramentos seja a ausência de germinação, uma vez que a temperatura nas rochas é maior do que no solo, limitando a germinação.

Geralmente, espécies pioneiras apresentam fotoblastismo positivo e alta produção de sementes pequenas e com alta longevidade (Souza \& Valio 2001). $\mathrm{Na}$ ausência de luz estas sementes podem ficar dormentes no solo e constituir os bancos de semente (Baider et al. 1999). Apesar das evidências de que M. taxifolia seja espécie pioneira, como outras Melastomataceae (Zaia \& Takaki 1998; Baider et al. 1999), estudos detalhados são necessários para averiguar se a pequena cobertura vegetal dos solos da Serra do Cipó, formada principalmente por gramíneas e ciperáceas (Giulietti et al. 1987), pode constituir barreira à entrada de luz, inibindo a germinação de M. taxifolia.

Esta informação é fundamental para sua utilização em programas de reabilitação ambiental, uma vez que M. taxifolia é potencialmente útil para este fim. Esta espécie é capaz de formar densas populações recobrindo efetivamente o solo, que rebrotam e crescem rapidamente após a ação do fogo (Vale, com. pess.). Os dados do presente trabalho são relevantes para subsidiar programas de reabilitação ambiental e conservação por meio da propagação por sementes de espécies nativas (ABNT 1998).

\section{Agradecimentos}

Os Autores agradecem a José Rubens Pirani, pela identificação da espécie; Luzia Márcia Araújo, pelo auxílio em laboratório; a Luzia Márcia Araújo, Fernando Henrique Aguiar Vale e dois revisores anônimos, pelos comentários e críticas em versões preliminares do artigo; ao CNPq (52 1772/95-8, 47.9684/2001-4), Fapemig (Cra 388/01, Cra 583/03) e Idea Wild Foundation, pelos auxílios concedidos.

\section{Referências bibliográficas}

ABNT - Associação Brasileira de Normas e Técnicas. 1998. Elaboração e apresentação de projetos de reabilitação de áreas degradadas pela mineração. NBR 13030, Brasil.

Andrade, A.C.S. 1995. Efeito da luz e da temperatura na germinação de Leandra breviflora Cogn., Tibouchina benthamiana Cogn., Tibouchina grandifolia Cogn., Tibouchina moricandiana (DC.) Baill. (Melastomataceae). Revista Brasileira de Sementes 17: 29-35.

Baider, C.; Tabarelli, M. \& Mantovani, W. 1999. O banco de sementes em um trecho de floresta Atlântica montana (São Paulo-Brasil). Revista Brasileira de Biologia 59: 319-328.

Baskin, C.C.; Baskin, J.M. \& Chester-Edward, W. 1999. Seed dormancy and germination in Rhexia mariana var. interior (Melastomataceae) and eco-evolutionary implications. Canadian Journal of Botany 77: 488-493.

Bewley, J.D. \& Black, M. 1994. Seeds: physiology of development and germination. Plenum Press, New York.

Costa, C.M.R.; Hermann, G.; Martins, C.G.; Lins, L.V. \& Lamas, I.R. (eds.). 1998. Biodiversidade em Minas Gerais. Fundação Biodiversitas, Belo Horizonte.

Garcia, Q.S. \& Diniz, I.S.S. 2003. Germinative behavior of three species of Vellozia from Serra do Cipó, MG. Acta Botanica Brasilica 17: 487-494.

Elisson, A.M.; Denslow, J.S.; Loiselle, B.A. \& Brenes, D.M. 1993. Seed and seedling ecology of neotropical Melastomataceae. Ecology 74: 1733-1749.

Felippe, G.M. \& Silva, J.C.S. 1984. Estudos de germinação em espécies do cerrado. Revista Brasileira de Botânica 7: 157-163.

Ferreira, A.G.; Cassol, B.; Rosa, S.G.T.; Silveira, T.S.; Stival. A.L. \& Silva, A.A. 2001. Germinação de sementes de Asteraceae nativas do Rio Grande do Sul, Brasil. Acta Botanica Brasilica 15: 231-242.

Giulietti, A.M.; Menezes, N.L.; Pirani, J.R.; Meguro, M. \& Wanderley, M.G.L. 1987. Flora da Serra do Cipó, Minas Gerais: caracterização e lista de espécies. Boletim de Botânica da Universidade de São Paulo 9: 1-151.

Gomes, V. \& Fernandes, G.W. 2002. Germinação de sementes de Baccharis dracunculifolia (Asteraceae). Acta Botanica Brasilica 16: 421-427.

Gomes, V.; Madeira, J.A.; Fernandes, G.W. \& Lemos Filho, J.P. 2001. Seed dormancy and germination of sympatric species of Chamaecrista (Leguminosae) in a rupestrian field. International Journal of Ecology and Environmental Sciences 27: 191-197.

Hewitt, N. 1998. Seed size and shade-tolerance: a comparative analysis of North American temperate trees. Oecologia 114: 432-440.

Jacobi, C.M.; Carmo, R. \& Oliveira, R. 2000. The reproductive biology of two species of Diplusodon Pohl (Lythraceae) from Serra do Cipó, southeastern Brazil. Plant Biology 2: $1-7$. 
Labouriau, L.G. 1983. A germinação das sementes. OEA, Washington.

Lara, A.C.F. \& Fernandes, G.W. 1996. The highest diversity of galling insects: Serra do Cipó, Brazil. Biodiversity Letters 3: 111-114.

Madeira, J.A. \& Fernandes, G.W. 1999. Reproductive phenology of sympatric Chamaecrista taxa of Chamaecrista (Leguminosae) in Serra do Cipó, Brazil. Journal of Tropical Ecology 15: 463-479.

Melo, J.T.; Silva, J.A.; Torres, R.A.A.; Silveira, C.E.S. \& Caldas, L.S. 1998. Coleta, propagação e desenvolvimento inicial de espécies do cerrado. Pp.195-246. In: S.M. Sano \& S.P. Almeida (orgs.). Cerrado: ambiente e flora. Embrapa, Planaltina.

Mendonça, M.P. \& Lins, L.V. (orgs.). 2000. Lista vermelha das espécies ameaçadas de extinção da flora de Minas Gerais. Fundação Biodiversitas, Fundação ZooBotânica de Belo Horizonte, Belo Horizonte.

Miranda, P.R.M. \& Ferraz, I.D.K. 1999. Efeito da temperatura na germinação de sementes e morfologia da plântula de Maquira sclerophylla (Ducke) C.C. Berg. Revista Brasileira de Botânica 22: 303-307.

Myers, N.; Mittermeier, R.A.; Mittermeier, C.G.; Fonseca, G.A.B. \& Kent, J. 2000. Biodiversity hotspots for conservation priorities. Nature 403: 853-858.
Ranieri. B.D.; Lana, T.C.; Negreiros, D.; Araújo, L.M. \& Fernandes, G.W. 2003. Germinação de sementes de Lavoisiera cordata e Lavoisiera francavillana (Melastomataceae), espécies simpátricas da Serra do Cipó, Brasil. Acta Botanica Brasilica 17: 523-530.

Sousa-Silva, J.C.; Ribeiro, J. F.; Fonseca, C.E.L. \& Antunes, N.B. 2001. Germinação de sementes e emergência de plântulas de espécies arbóreas e arbustivas que ocorrem em Matas de Galeria. Pp. 379-422. In: J.F. Ribeiro; C.E.L. Fonseca \& J.C. Sousa-Silva. Cerrado: Caracterização e recuperação de Matas de Galeria. Embrapa, Planaltina, DF.

Souza, R.P. \& Valio, I.F.M. 2001. Seed size, seed germination and seedling survival of Brazilian tropical tree species differing in successional status. Biotropica 33: 447-457.

Valio, I.F.M. \& Scarpa, F.M. 2001. Germination of seeds of tropical pioneer species under controlled and natural conditions. Revista Brasileira de Botânica 24: 79-84.

Vásques-Yanes, C. \& Orozco-Segovia, A. 1993. Patterns of seed longevity and germination in the tropical rain forest. Annual Review of Ecology and Systematics 24: 69-87.

Zaia, J.E. \& Takaki, M. 1998. Estudo da germinação de sementes de espécies arbóreas pioneiras: Tibouchina pulchra Cogn e Tibouchina granulosa Cogn. Acta Botanica Brasilica 12: 227 -238.

Zar, J.H. 1996. Biostatistical analysis. 3 ed. Prentice Hall, New Jersey. 